\section{(O) OPEN ACCESS}

\title{
Human placenta as an ex vivo vascular model for neurointerventional research
}

\author{
John Ching Kwong Kwok, ${ }^{1,2}$ Wei Huang, ${ }^{3}$ Wing-Cheong Leung, ${ }^{4}$ Siu-Ki Chan, ${ }^{5}$ \\ Kwong-Yau Chan, ${ }^{1}$ Kar-Ming Leung, ${ }^{1}$ Alberto Chi Ho Chu, ${ }^{1}$ Alexander Kar Ngai Lam ${ }^{3}$
}

\begin{abstract}
${ }^{1}$ Department of Neurosurgery, Kwong Wah Hospital, Kowloon, Hong Kong ${ }^{2}$ Division of Biomedical Engineering, Hong Kong University of Science and Technology, Kowloon, Hong Kong ${ }^{3}$ Department of Mechanical Engineering, Hong Kong University of Science and Technology, Kowloon, Hong Kong

${ }^{4}$ Department of Obstetrics and Gynecology, Kwong Wah Hospital, Kowloon, Hong Kong ${ }^{5}$ Department of Pathology, Kwong Wah Hospital, Hong Kong, Kowloon, Hong Kong
\end{abstract}

\section{Correspondence to} Dr J C K Kwok, Department of Neurosurgery, Kwong Wah Hospital, 25 Waterloo Road, Kowloon, Kowloon, Hong Kong; jckkwok@ha.org.hk

Received 6 May 2013 Revised 3 July 2013 Accepted 5 July 2013 Published Online First 31 July 2013

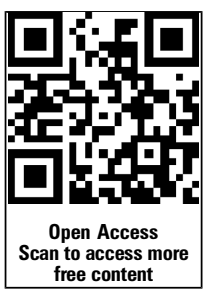

To cite: Kwok JCK, Huang W, Leung W-C, et al. I Neurolntervent Surg 2014;6:394-399.

\section{ABSTRACT}

Background Human placenta is a convenient resource for biomedical research, and has not yet been used for neurointerventional surgery research.

Objective Our objective was to explore the feasibility of using human placenta to test various endovascular interventions and for training.

Design 18 placentas soon after delivery were prepared for six pilot studies. (1) Study on anatomical similarity to human cerebral vessel. (2) Simulation of stent assisted coiling and flow diversion on an aneurysm model. (3) Simulation of intra-arterial thrombolysis. (4) Simulation of embolization of arteriovenous malformation with glues.

(5) Simulation of mechanical thrombolysis and comparison of different devices. (6) Vascular model for training of neurointerventionalists.

Results When the chorionic plate vessels were compared with the cerebral cortical vessels, similarities were found in vascular branch patterns, histological cross sections, and angiographic appearances. Due to the semitransparency of its vessel wall, performance of flow diverter and stent assisted coiling of an aneurysm could be visualized under direct microscopic observation. Similarly, timing of clot lysis and glue polymerization could be estimated. Endothelial change after thrombectomy could be assessed by histological methods. From these pilot studies, the placenta model could be adopted to simulate various clinical situations. It is also ideal for interventional radiology training. Conclusions It is feasible to adopt the human placenta as an ex vivo vascular model in neurointerventional surgery research due to the fact that its vessels resemble the brain vasculature.

\section{INTRODUCTION}

Endovascular therapy for cerebral vascular diseases has expanded in both the variety and number of devices. Conventionally, an animal vascular model is used for initial testing of new devices. ${ }^{12}$ To date, swine and $\operatorname{dog}$ models have been reported. ${ }^{3}$ However, animal experiments are labor intensive and expensive. Fresh human placenta on the other hand is relatively easy to obtain from hospitals with obstetrics service. Although the placenta has been widely used in biomedical research, ${ }^{4} 5$ its application in neurointerventional research has not been found in the literature. We now report on the preliminary applications of human placenta as an ex vivo vascular model in this rapidly expanding research field.

\section{METHODS}

Written consent for donation of placenta for research was obtained from the mother before the baby was delivered. Eighteen placentas were obtained during the period 2011-2013 for various vascular interventions. Fresh placentas soon after delivery were used. After cleansing with normal sterile saline solution to remove any blood adhered to the placenta surface, the amnion sac was peeled off from the chorion and removed by sharp dissection at the cord insertion, thus exposing the chorionic plate vessels on the chorion surface. The umbilical cord was shortened to about $10 \mathrm{~cm}$ to allow easy catheterization of the umbilical arteries and vein. A 5 French gauge straight tip diagnostic catheter or size 16 venous puncture catheter was used to catheterize the umbilical arteries. A pressurized intra-arterial infusion bag was used to delivery heparinized saline solution into the placental arteries (figure 1A). A motorized pump was used to simulate pulsation of the vessels. A solution of heparin in normal saline was used to irrigate the specimen until the vessels were free of blood clots. By taking advantage of the semitransparent nature of the placental vessels, direct observations of changes within the blood vessels and the behavior of catheters, clot and devices under a Leica OH5 neurosurgical microscope (Wetzlar, Germany) with high magnification, were made. Results were documented by high definition digital recording and photography. By erecting the placenta with a Styrofoam board support on a tray, digital subtraction angiography (DSA) was performed (figure 1B). This set-up was to allow escape of fluid and contrast material from the placenta to the tray by gravity, thus keeping the radiological field clear (figure 1C). A strict transmissible disease protection protocol was observed in all procedures.

Six experimental studies were performed. (1) Study on anatomical similarity to human cerebral vessel. (2) Simulation of stent assisted coiling and flow diversion on an aneurysm model. (3) Simulation of intra-arterial thrombolysis. (4) Simulation of embolization of arteriovenous malformation (AVM) with glues. (5) Simulation of mechanical thrombolysis and comparison of different devices. (6) Vascular model for training of neurointerventionalists.

All 18 placentas were examined macroscopically and the diameters of the first order chorionic plate artery (CPA) and vein were recorded. The presence of any anastomosis between the two umbilical arteries near the placental insertion was noted. The specimens were divided into six groups for the pilot studies. 


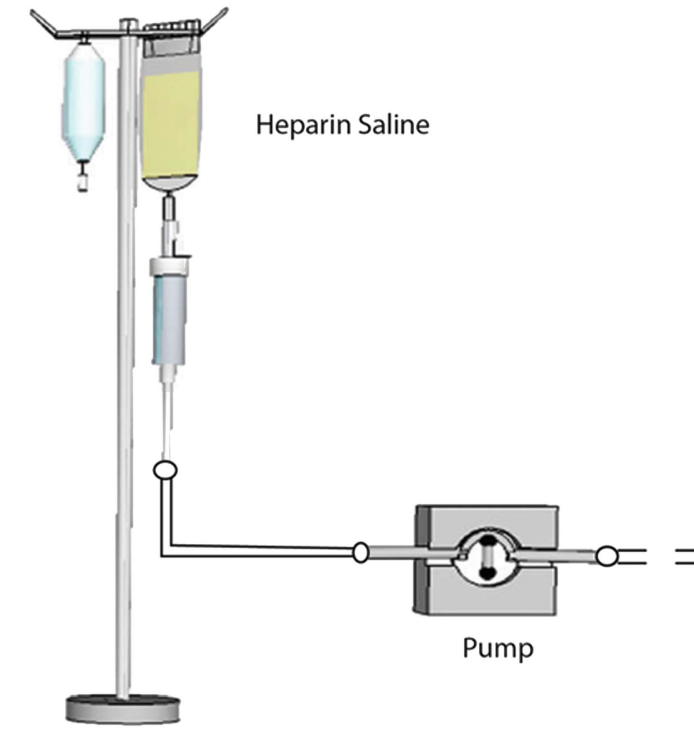

A

Figure 1 (A) Illustration of the placenta vascular model experimental set-up. DSA, digital subtraction angiography. (B) Mounting of the placenta for DSA. (C) Angiographic appearance of the chorionic plate arteries and venous drainage.

For experimental study Nos 1, 2, 3, 4, and 5, a neurosurgical microscope was used for microdissection, visualization of flow inside the vessel, and high definition photography. In experimental study No 3, ex vivo intra-arterial thrombolysis was performed with 500000 units of urokinase. With the use of a pulsatile pump, the placenta was continuously perfused with Ringer's solution via a 6 French gauge catheter to simulate a normal circulation (figure 1A). A $5 \mathrm{~mm}$ in length with $4 \mathrm{~mm}$ diameter clot specimen was loaded into the junction of the first and second order arteries through the guiding catheter. Under high magnification, the lytic change of the clot sample was observed through the almost transparent CPA.

In experimental study No 5, different thrombectomy devices were tested for ease of deployment and effectiveness in clot retrieval en masse. After single passage of the device, the labeled vessel was fixed in situ by vascular perfusion of $4 \%$ formaldehyde in normal saline for $60 \mathrm{~min}$ under a perfusion pressure of $50 \mathrm{~mm} \mathrm{Hg}$ and a draining pressure of $0 \mathrm{~mm} \mathrm{Hg}$ at the placental capillaries region. The vascular samples were dissected and prepared for paraffin sections and en face endothelium examination. The paraffin sections were performed with hematoxylin-eosin stain to visualize the vessel morphology and cluster of differentiation 31 (CD31) immunohistological stain to confirm the existence of endothelium, and were then examined by microscopy. For en face examination, the tissue samples were incubated with a mixture of $5 \mathrm{Unit} / \mathrm{mL}$ Alexa Fluor 488 phalloidin (Invitrogen, Camarillo, California, USA), 1\% bovine serum albumin (Santa Cruz Biotechnology, Santa Cruz, California, USA), 0.5\% Triton X-100 (Invitrogen), and physiological buffered saline for $1 \mathrm{~h}$ at $37^{\circ} \mathrm{C}$. After rinsing three times in physiological buffered saline for 5 min each time, the samples were stained with a 1:500 dilution of To-Pro-3 (Invitrogen) for $20 \mathrm{~min}$ at room temperature. Tissue samples without phalloidin and To-Pro-3 treatments were used as controls. The fluorescence stained samples were placed on Superfrost Plus Gold slides with the endothelium facing upward, and then mounted with Pro-Long Gold antifade reagent (Invitrogen). After covering with a micro glass and the edges sealed, the slides were examined with a LSM 7 Duo confocal microscope (Carl Zeiss, Jena, Germany).

Experimental study Nos 4 and 6 were performed under DSA. The placenta was perfused with dextrose non-ionic solution before the application of N-butylcyanoacrylate (NBCA)/ethiodized oil (Lipiodol) mixture; 18\% NBCA was used to visualize the angiographic appearance and polymerization time. Ethylene 
vinyl alcohol copolymer in dimethyl sulfoxide (Onyx) was tested for the degree of penetration and reflux. After DSA examination, the specimens were examined under direct microscopy.

\section{RESULTS}

\section{Study No 1}

The primary branches of the CPA of all 18 placentas were measured. The average diameter was $4.2 \mathrm{~mm}$. They have the size of the average intracranial internal carotid artery. The cerebral cortical veins on the brain surface always cross over the arteries (figure 2A) while the CPAs always cross over veins when observed from the fetal surface (figure 2B). Similarities were found in vascular branch patterns and histological cross sections of the human cerebral artery (figure 2C,E) and the CPA (figure 2D,F). A single anastomosis between umbilical arteries at cord insertion was detected in all specimens.

\section{Study No 2}

Intracranial stents such as Pipeline, Neuroform, and Wingspan stents (Covidien, USA) were performed with the use of a delivery catheter. One of the main side branches of the placenta vessel was ligated to simulate a wide neck aneurysm (figure $3 \mathrm{~A}$ ). In one model, anticoagulated human blood was infused into a Pipeline mounted vessel with side branch ligated. Flow diversion was observed with examination of the interface between the Pipeline device and the aneurysm (figure 3B). Similarly, deployment of coil through the stent strut into the aneurysm was done. Under magnification, shape conformation of the coil during detachment by electrolysis or mechanical means was visualized.

\section{Study No 3}

The dose of lytic agent was diluted to $10 \mathrm{~mL}$, and the time to complete lysis of the clot was 23 min on average (figure $3 \mathrm{C}, \mathrm{D}$ ).

\section{Study No 4}

Three placentas were used for glue embolization. The DSA appearance resembled embolization of the AVM feeding vessel (figure 3E,F). The perforating branches of the placenta vessel are very similar to the en passage configuration of AVM. The polymerization times for both glues were similar to the clinical

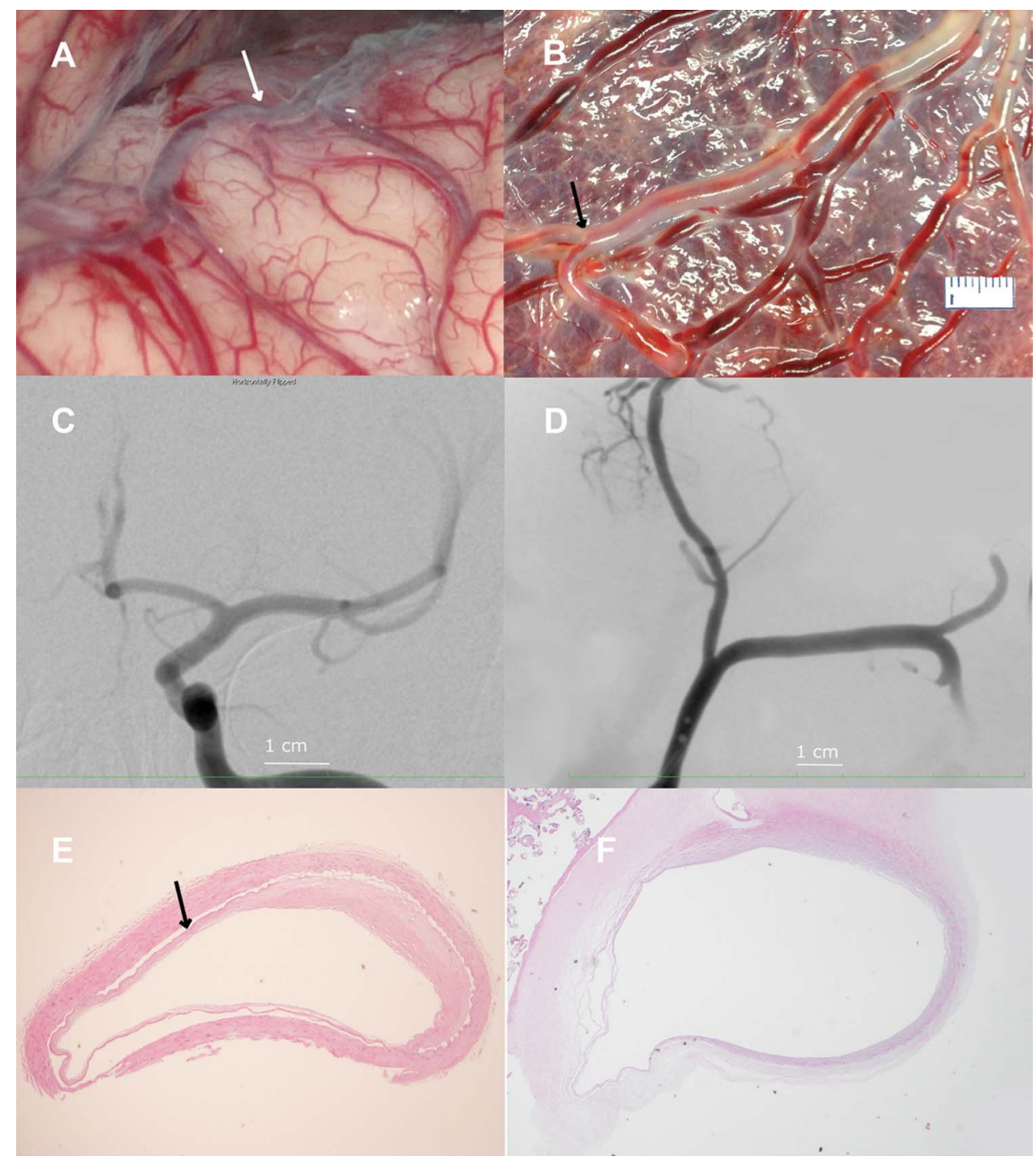

Figure 2 (A) Brain surface at the region of the Sylvian fissure where the Sylvian vein crosses the temporal branch of the middle cerebral artery (white arrow). The cerebral vein is superficial to the artery. (B) Chorionic surface after removal of the amnion membrane showing the chorionic plate artery (black arrow) crossing the vein. (C) Digital subtraction angiography (DSA) of the left internal carotid artery (ICA) in a human subject. (D) DSA of the chorionic plate arteries in a human placenta. (E) Hematoxylin-eosin (H\&E) staining of a cross section of the human ICA showing the presence of the tunica elastica (black arrow). (F) H\&E staining of a cross section of the chorionic plate artery showing the absence of the tunica elastica. 


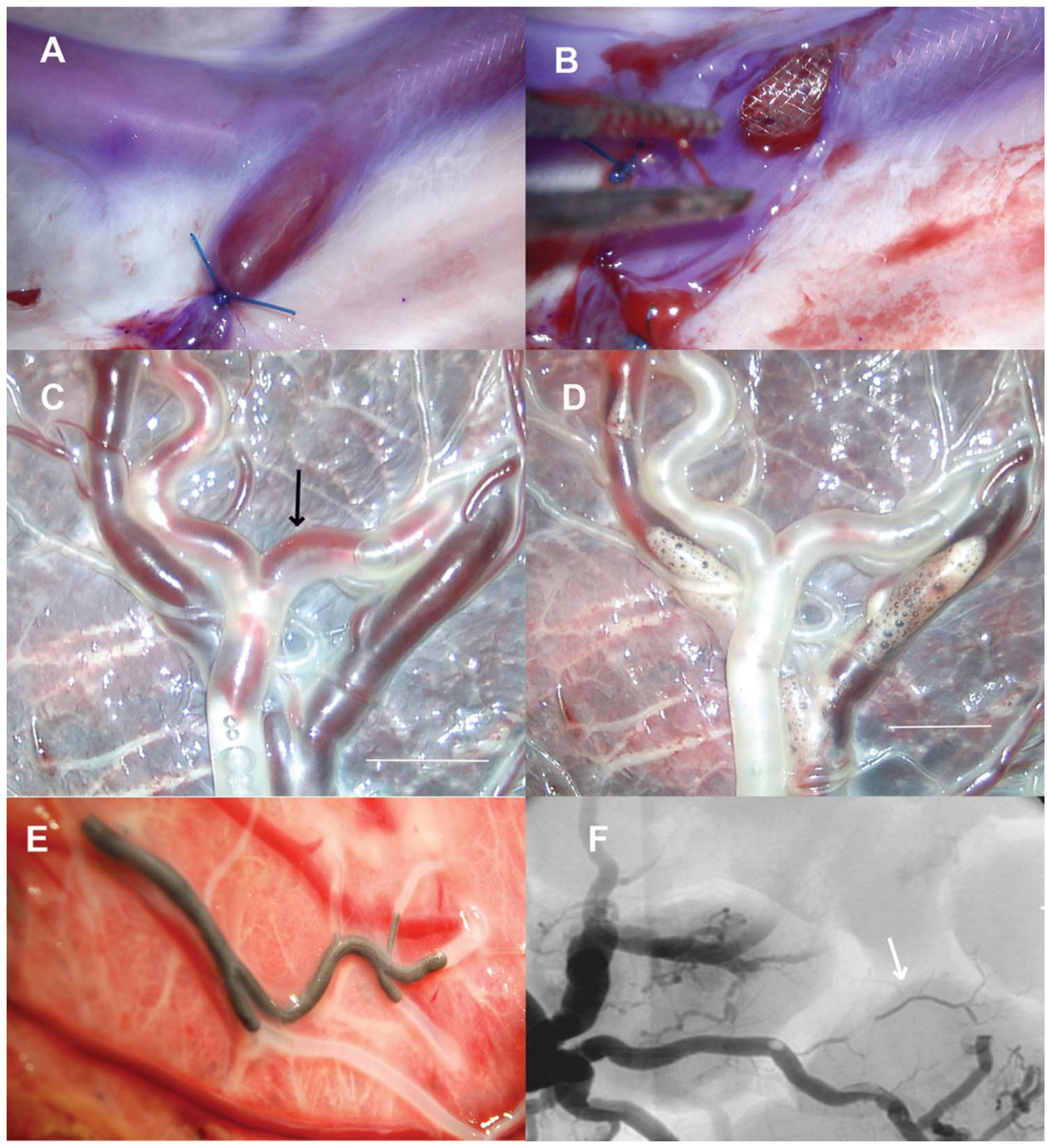

Figure 3 (A) Simulation of flow diversion of a wide neck aneurysm by the pipeline device (PD). A side branch of the chorionic plate artery (CPA) was ligation to form an aneurysm-like sac. A heparinized human blood sample was perfused into the vessel for 30 min. (B) The vessel was opened to inspect the surface of the PD and clot formation. (C) Simulation of intra-arterial thrombolysis with clot sample (black arrow) was loaded into the bifurcation of a CPA. (D) The clot was completely lysed in 23 min by urokinase. (E) Appearance of Onyx embolization at the distal CPA. (F) Digital subtraction angiography appearance of the embolized vessel (white arrow). Degree of adhesiveness of Onyx with the detachable tip of the perfusion catheter was observed.

situation even when the placentas were perfused with dextrose solution instead of fresh blood.

\section{Study No 5}

Deployment of devices such as the Penumbra Separator 3D (Penumbra Europe GmbH, Germany), Solitaire FR stent (Covidien), and similar stent-like constructs were performed in a preselected branch of the placenta artery under continuous Ringer's solution perfusion (figure 4A,B). After applying single passage with a thrombectomy device, the blood vessels were removed from the placenta and histological examinations performed. The change in endothelium lining after thrombectomy was compared with the normal control. The hematoxylin-eosin staining method demonstrated the vessel wall layers (figure 4C). Since the endothelium of CPA was positive for the CD31 stain (figure 4D), the change in endothelial lining could be compared for two different devices after thrombectomy at the same placenta. The change was confirmed by confocal microscopy and quantitative measurement of cell loss (figure 4E,F). This initial study was affirmative of endothelium loss after the use of the thrombectomy device. No concrete conclusion could be drawn from these findings as the number of vessels examined was too small for statistical analysis.

\section{Study No 6}

Three neurosurgical trainees participated in this study and performed DSA by catheterizing the umbilical artery or vein at the loose end of the umbilical cord. Various modes of angiographic examination were performed, such as hand injection of contrast or glue, or deployment of stent or thrombectomy devices. The placental model was well suited for training of pushing NBCA glue and withdrawal of a delivery catheter, which require multiple practices to achieve ideal results in that split second manipulation.

\section{DISCUSSION}

Although the placenta shows a wide variation in its anatomy between individuals, in the majority of the cases it is perfused with blood by two umbilical arteries and one vein. The blood vessels spread and divide in the two-dimensional plane between the amnion and the chorion on the fetal side of the placenta, constituting the CPAs. The CPAs form a disperse pattern where they divide dichotomously, in two equal branches, with gradually 

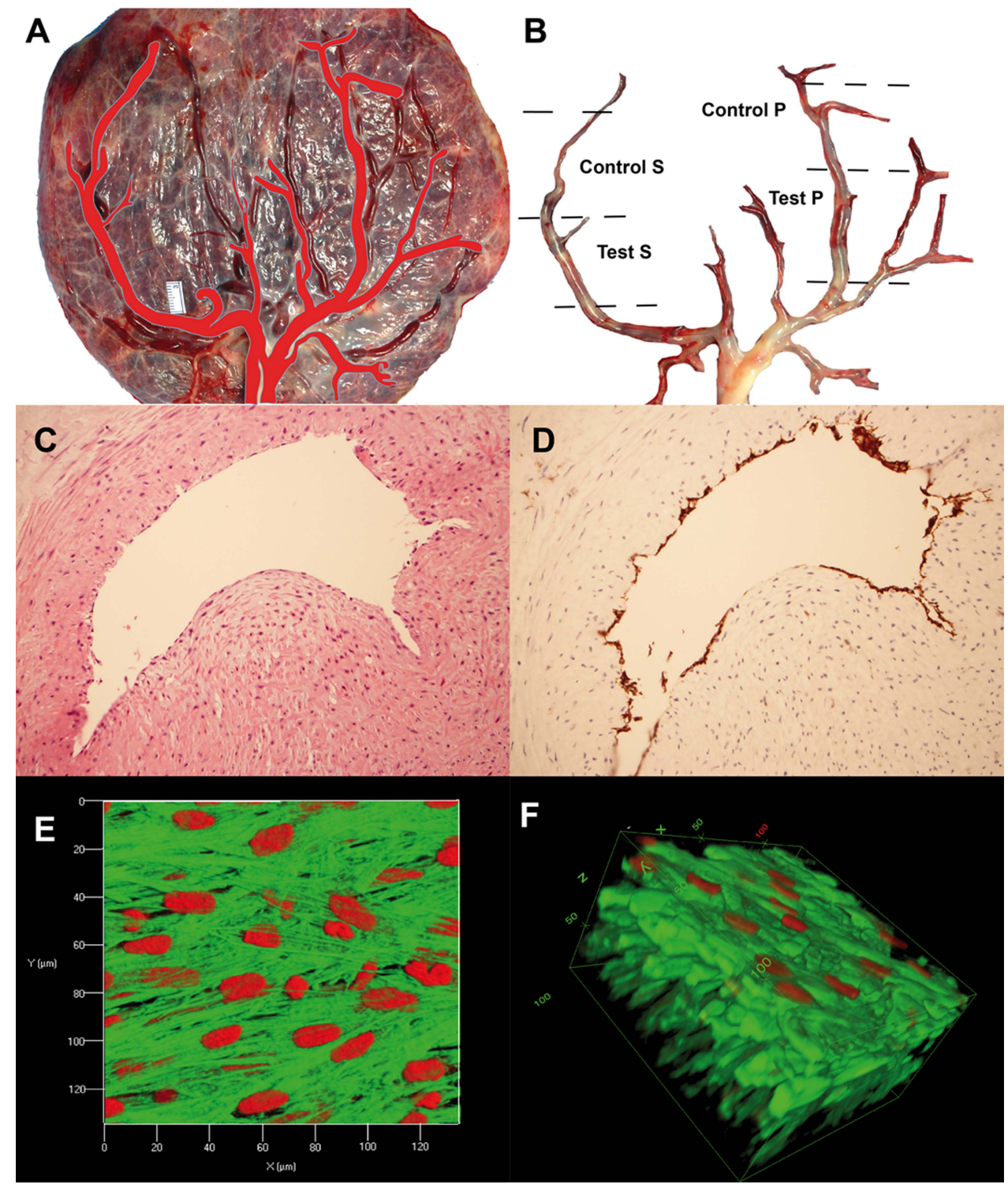

Figure 4 (A) Image tracing of the chorionic plate artery (CPA) on the surface of the placenta. (B) Mapping of the CPA with designation of control and testing segments for comparing embolectomy devices. (C, D) Cross sectional images of a CPA obtained by light microscopy. The tissue samples were stained with hematoxylin-eosin and CD31 for endothelium, respectively. (E) Optical section two-dimensional images at $1 \mu \mathrm{m}$ showing endothelial cell nuclei. (F) A three-dimensional image was constructed with a series of optical section two-dimensional images along the thickness of the vessel wall ( $\mathrm{z}$ axis).

diminishing diameter, giving off small branches along their course. The primary branches of the umbilical vasculature that course through the chorionic plate periodically dive beneath this stratum to establish the circulation of primary vascular ramifications ending in the terminal villi. ${ }^{6}$ The two dichotomized branches are extremely useful in adapting them for comparison of two devices or using one as control on the same placenta.

Resembling the cortical blood vessels of the human brain, the CPAs and their branches are end arteries. ${ }^{7}$ The veins roughly follow the course of the arteries, showing a similar branching pattern. The chorionic plate veins carry oxygenated blood, similar to the cerebral arteries. There are two significant differences however. Firstly, the blood pressure difference between the cerebral artery and vein is more than $100 \mathrm{~mm} \mathrm{Hg}$ while between the chorionic artery and vein it is only $30 \mathrm{~mm} \mathrm{Hg}$ under normal physiological conditions. Due to different pressure requirements, the properties and thickness of the vessel wall between the cerebral artery and vein are distinctly different while the chorionic vessels show less disparity. As a result, both placental artery and venous systems can be used to simulate the cerebral artery whereas the animal model is limited to one. Secondly, the chorionic artery is superficial to the vein while the cerebral cortical artery is the reverse. As the chorionic artery tree is superficial, movement of materials inside the vessel lumen can be visualized throughout the entire length without disruption. The differences are adding merits to the model rather than discrediting it. Further similarity was found in the covering of the organ. There are four extraembryonic membranes that are part of the placenta, including the yolk sac, amnion, allantois, and chorion. For the brain, there are also four layers of membrane coverings-the fibrous dura, meningeal dura, arachnoid, and pia matter. In most placentas there is a single anastomosis, first described by Hyrtl in 1870, between the umbilical arteries near the cord insertion. ${ }^{8}$ The anastomosis is promising for simulation of communication between the left and right cerebral vascular territories. Further experiment is required to validate the usefulness of this model for cross flow study. 
Freshly delivered placenta is carrying living cells. Cooling and irrigation with iso-osmotic fluid such a Ringer's solution can preserve the tissues. We found that the cells within the blood vessels of the human placenta in particular were still viable to take up vital staining for histological studies within $6 \mathrm{~h}$ after placenta delivery (figure 4). CD31 is a constituent of the endothelial intercellular junction, also designated as PECAM-1 (platelet endothelial cell adhesion molecule 1). ${ }^{9}$ Immunohistological CD31 stain has been applied to routinely processed tissues containing endothelial cells. ${ }^{10}$ Phalloidin binds F-actin with high selectivity, and has been used to visualize and quantify F-actin in tissue sections and cell cultures. ${ }^{11}$ The To-Pro-3 stain is a sensitive detector of double stranded nuclei acids. ${ }^{12}$ It may be worthwhile exploring the applications of molecular markers in the ex vivo model.

Most of the placentas demonstrated various degrees of chorionic plate vessel spasm soon after delivery. The smooth muscle constriction may be triggered by the release of angiotensin II from the placenta. ${ }^{13}$ In our observation, the pressurized infusion of Ringer's solution for 10-15 min can also restore the normal luminal size of the vessels. After the application of heparin and urokinase infusion, the existing clot within the arteries can be dissolved and passed to the venous system of the placenta. The only difference is the presence of the autonomic nervous supply to the smooth muscle layer in the cerebral blood vessel where the placenta is deprived of any nerve supply. With general features closely resembling the brain vessel in terms of diameter, tortuosity, elasticity, and bifurcation pattern at the chorion surface, the human placenta is well suited as an ex vivo vascular model to simulate various clinical situations.

Interventional procedures are well known to carry a high risk of procedure related complications, such as inadvertent perforation of a vessel, kinking of a catheter or guidewire, fracture of catheters, and overshooting of embolization materials. For trainees, apart from computer simulation training, the placental model can provide start up practice in the DSA room before they embark on performing interventional procedures in real clinical situations.

\section{CONCLUSION}

The experimental studies in this paper suggest that it is feasible to use human placenta as an ex vivo vascular model in neurointerventional surgery research and training purposes due to its vessels resembling the brain vasculature. The model opens doors for investigation of new endovascular devices during the development phase in the future.

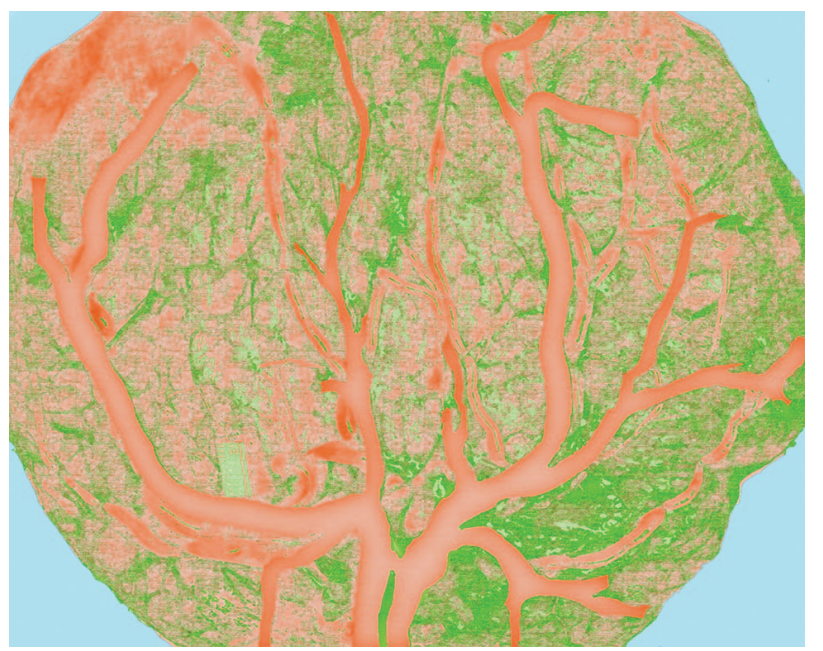

'Tree of life'-modified graphic of the placental vasculature.
Acknowledgements We would like to thank the staff of the Department of Obstetrics and Gynecology, Kwong Wah Hospital, for their assistance in securing the placenta specimens for this study.

Contributors JCKK: guarantor, conception of the study, design of experiment Nos $1,3,4$, and 6 , drafting of the article, and final approval of the version to be published. WH: design of the confocal microscopy, data collection, drafting of the article, and final approval of the version to be published. W-CL: placenta anatomical comparison, drafting of the article, and final approval of the version to be published S-KC: histological experimental design and comparison, drafting of the article, and final approval of the version to be published. K-YC and K-ML: conception of the study, design of experiment Nos 5 and 6 and interpretation of radiological results, revision of the article, and final approval of the version to be published. ACHC and AKNL: acquisition of the data, design of experiment No 2, revision of the article, and final approval of the version to be published.

Funding This study was supported by a generous donation from Dr Simon Kwok and Dr Eleanor Kwok.

\section{Competing interests None.}

Consent Written consent for donation of placenta from the mothers was obtained. A full explanation of the use of the placenta for research in a printed format was provided to the mothers.

Provenance and peer review Not commissioned, externally peer reviewed.

Data sharing statement Additional photographic documentation of the experiments and model are available on request to the corresponding author.

Open Access This is an Open Access article distributed in accordance with the Creative Commons Attribution Non Commercial (CC BY-NC 3.0) license, which permits others to distribute, remix, adapt, build upon this work non-commercially, and license their derivative works on different terms, provided the original work is properly cited and the use is non-commercial. See: http://creativecommons.org/ licenses/by-nc/3.0/

\section{REFERENCES}

1 Yuki I, Kan I, Vinters HV, et al. The impact of thromboemboli histology on the performance of a mechanical thrombectomy device. AJNR Am J Neuroradiol 2012:33:643-8

2 Roth C, Junk D, Papanagiotou P, et al. A comparison of 2 stroke devices: the new Aperio clot-removal device and the solitaire AB/FR. AJNR Am J Neuroradiol 2012;33:1317-20.

3 Bacigaluppi M, Comi G, Hermann DM. Animal models of ischemic stroke. Part two: modeling cerebral ischemia. Open Neurol J 2010;4:34-8.

4 Myllynen $\mathrm{P}$, Pasanen M, Pelkonen 0. Human placenta: a human organ for developmental toxicology research and biomonitoring. Placenta 2005;26:361-71.

5 Hutson JR, Garcia-Bournissen F, Davis A, et al. The human placental perfusion model: a systematic review and development of a model to predict in vivo transfer of therapeutic drugs. Clin Pharmacol Ther 2011;90:67-76.

6 Kaufmann P. Basic morphology of the fetal and maternal circuits in the human placenta. Contrib Gynecol Obstet 1985;13:5-17.

7 Duvernoy HM, Delon S, Vannson JL. Cortical blood vessels of the human brain. Brain Res Bull 1981;7:519-79.

8 Ullberg U, Sandstedt B, Lingman G. Hyrtl's anastomosis, the only connection between the two umbilical arteries. A study in full term placentas from AGA infants with normal umbilical artery blood flow. Acta Obstet Gynecol Scand 2001;80:1-6.

9 Miettinen $\mathrm{M}$, Lindenmayer AE, Chaubal A. Endothelial cell markers CD31, CD34, and $\mathrm{BNH} 9$ antibody to $\mathrm{H}$ - and $\mathrm{Y}$-antigens-evaluation of their specificity and sensitivity in the diagnosis of vascular tumors and comparison with von Willebrand factor. Mod Pathol 1994;7:82-90.

10 Pusztaszeri MP, Seelentag W, Bosman FT. Immunohistochemical expression of endothelial markers CD31, CD34, von Willebrand factor, and Fli-1 in normal human tissues. J Histochem Cytochem 2006;54:385-95.

11 Adams AE, Pringle JR. Staining of actin with fluorochrome-conjugated phalloidin. Methods Enzymol 1991;194:729-31.

12 Bink K, Walch A, Feuchtinger A, et al. TO-PRO-3 is an optimal fluorescent dye for nuclear counterstaining in dual-colour FISH on paraffin sections. Histochem Cell Biol 2001;115:293-9.

13 Benoit C, Gu Y, Zhang Y, et al. Contractility of placental vascular smooth muscle cells in response to stimuli produced by the placenta: roles of ACE vs. non-ACE and AT1 vs. AT2 in placental vessel cells. Placenta 2008;29:503-9. 